Planetary Systems in the Universe - Observation, Formation and Evolution

Proceedings IAU Symposium No. 202, (c)2004 IAU

Alan Penny, Pawel Artymowicz, Anne-Marie Lagrange, 8 Sara Russell, eds.

\title{
Planet Detection Capabilities of the Eddington Mission
}

\author{
Hans J. Deeg \\ Instituto de Astrofísica de Andalucía, Granada, Spain; hdeeg@iaa.es \\ Instituto de Astrofísica de Canarias, Tenerife, Spain \\ Keith Horne \\ St.Andrews University, School of Physics and Astronomy, North Haugh, \\ St. Andrews, Fife KY16 9SS, Scotland, UK
}

Fabio Favata and the Eddington Science Team (C. Aerts, E. Antonello, M. Badiali, C. Catala, J. Christensen-Dalsgaard, A. Gimenez, M. Grenon, A. Penny, H. Rauer, I.W. Roxburgh, J. Schneider, N.R. Waltham)

\begin{abstract}
Astrophysics Division - Space Science Department of ESA, ESTEC, Postbus 299, NL-2200 AG Noordwijk, The Netherlands
\end{abstract}

\begin{abstract}
Eddington is a space mission for extrasolar planet finding and for asteroseismic observations. It has been selected by ESA as an F2/F3 reserve mission with a potential implementation in 2008-13. Here we describe Eddington's capabilities to detect extrasolar planets, with an emphasis on the detection of habitable planets. Simulations covering the instrumental capabilities of $E d$ dington and the stellar distributions in potential target fields lead to predictions of about 10,000 planets of all sizes and temperatures, and a few tens of terrestrial planets that are potentially habitable. Implications of Eddington for future larger scale missions are briefly discussed.
\end{abstract}

\section{The Eddington mission}

The Eddington mission is a space telescope designed for two primary goals: asteroseismic studies and extrasolar planet finding. Both goals will be achieved through the acquisition of high-precision wide-field photometry, with a temporal stability that is possible only from space. The basic design is an $f / 3$ triplereflecting telescope with a $1.2 \mathrm{~m}$ diameter aperture, $0.6 \mathrm{~m}^{2}$ effective area, and a $3^{\circ}$ diameter field of view imaged by a 20-CCD mosaic camera. The telescope will be launched into an orbit around the L2 Earth-Sun libration point, from which the entire sky is accessible for observations. The first 2 years of the mission will be dedicated primarily to asteroseismic studies, with pointings lasting up to two months to a variety of targets. The mission's second part will be dedicated to planet finding by the transit method, where the telescope will survey a single stellar field for 3 years. In total, about 500,000 stars will be surveyed by Eddington as potential hosts for planetary systems. 

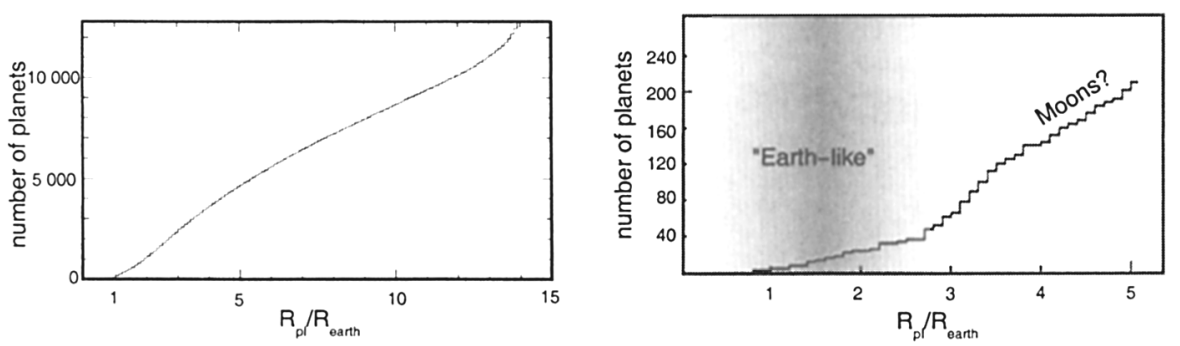

Figure 1. Expected count of detected planets. Left: all planets at any temperature or distance from the central star. Right: planets in the 'habitable zone' $(250-350 \mathrm{~K})$. The field 'Earth-like' indicates the size range of planets suitable to support life (solid surface and ability to maintain atmosphere). Moons around larger planets may also indicate life sites.

In October 2000, Eddington was selected by ESA as a reserve mission for the F2/F3 launch window (2009-2013). More details about the mission can be found in the Eddington Assessment study (Favata et al., 2000) and in the Eddington web-site (http://astro.esa.int/SA-general/Projects/Eddington/). Here we will give a short overview about its planet detection capabilities.

\section{Planet detections}

The evaluation of Eddington's planet detection performance consisted of two parts: First, we determined the kinds of planets this mission can detect, in terms of the planet's size, orbital period, and magnitude and type of central star (Deeg et al. 2000). Second, the numbers and characteristics of the detected planets were estimated. This employed Monte-Carlo simulations to fold Eddington's 'detection space' with models of the Galactic stellar distribution, density and scale height (Robin \& Crézé, 1986). With plausible exo-planet distributions, some 10,000 of planets of any size and orbital distance may be detected (Fig 1 , left). Of the detected planets the vast majority are both large and in close orbits around their central stars. The most extreme ones, the 'Hot Giants', will be detected not only by transits, but also by the reflected light of their central star (even if they do not produce transits) yielding a large and nearly complete sample of these planets. For smaller planets there are fewer sufficiently bright host stars within the observational range, and they will be detected only by transits. The habitable zone corresponds roughly to orbital distances at which planetary surface temperatures of $250-350 \mathrm{~K}$ are expected. Under the assumption that every main-sequence star has one planet in the habitable zone, a few hundred planets in that zone should be detected, among them a few tens of habitable planets with sizes of less then $2.5 R_{E a r t h}$, which should have solid surfaces (Fig 1, right). Around larger planets within the habitable zone there exists the intriguing possibility that massive moons may be detected through the perturbations they cause on the arrival time of transit minima (Sartoretti $\&$ Schneider, 1999). These moons may themselves be harboring sites for life. 


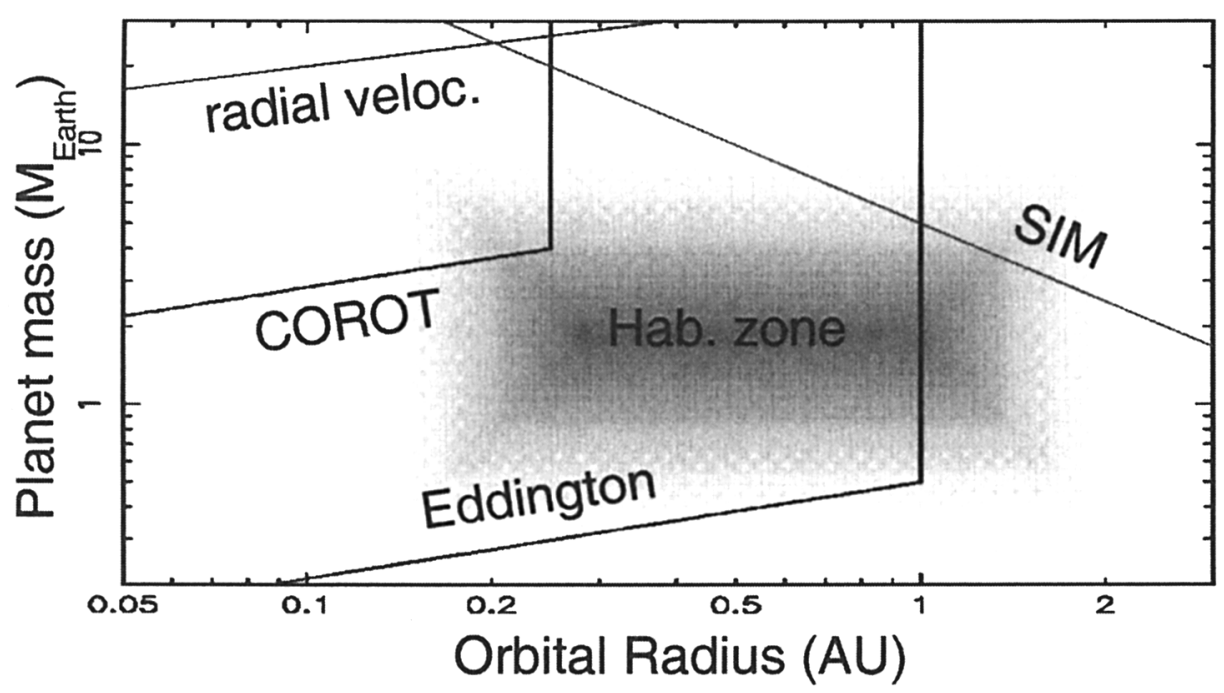

Figure 2. Comparison of planet finding capabilities of several missions. SIM's sensitivity is given for a star at 5pc distance. The limits for groundbased radial velocity searches are for a sensitivity of $1 \mathrm{~m} / \mathrm{s}$

\section{Eddington in perspective}

As Fig. 2 shows, Eddington is the first mission that will survey the habitable zone, and deliver a broad overview of the abundance of habitable planets in the Galaxy. This is an important step beyond missions like COROT and SIM, which may detect a few special cases of habitable planets. Eddington will deliver a large well-defined statistical sample of planets with known planet radii, orbital distances, and host star surface temperature and type. This is critical input for the design and target selection of future missions like Darwin and TPF that aim for detailed characterizations of Earth-like planets. Eddington moves us one step closer to resolving the age-old question: Are there other worlds like ours?

\section{References}

Deeg H.J., Favata F., and Eddington Science Team 2000, in 'Disks, Planetesimals and Planets', Tenerife, Eds. F. Garzon et al., ASP Conf proc., in print. Preprint astro-ph/0006130

Favata F., Roxburgh I., Christensen-Dalsgaard J. (Eds.) 2000, 'Eddington Assessment Study Report', ESA report ESA-SCI(2000)8

Robin A., Crézé M. 1986, A\&A 157, 71

Sartoretti P., Schneider J. 1999, A\&AS 234, 553 